\title{
Independent absorbed-dose calculation using the Monte Carlo algorithm in volumetric modulated arc therapy
}

Akihiro Haga*, Taiki Magome, Shigeharu Takenaka, Toshikazu Imae, Akira Sakumi, Akihiro Nomoto, Hiroshi Igaki, Kenshiro Shiraishi, Hideomi Yamashita, Kuni Ohtomo and Keiichi Nakagawa

\begin{abstract}
Purpose: To report the result of independent absorbed-dose calculations based on a Monte Carlo (MC) algorithm in volumetric modulated arc therapy (VMAT) for various treatment sites.

Methods and materials: All treatment plans were created by the superposition/convolution (SC) algorithm of SmartArc (Pinnacle V9.2, Philips). The beam information was converted into the format of the Monaco V3.3 (Elekta), which uses the X-ray voxel-based $M C(X V M C)$ algorithm. The dose distribution was independently recalculated in the Monaco. The dose for the planning target volume (PTV) and the organ at risk (OAR) were analyzed via comparisons with those of the treatment plan.

Before performing an independent absorbed-dose calculation, the validation was conducted via irradiation from 3 different gantry angles with a $10-\times 10-\mathrm{cm}^{2}$ field. For the independent absorbed-dose calculation, 15 patients with cancer (prostate, 5; lung, 5; head and neck, 3; rectal, 1; and esophageal, 1) who were treated with single-arc VMAT were selected. To classify the cause of the dose difference between the Pinnacle and Monaco TPSs, their calculations were also compared with the measurement data.

Result: In validation, the dose in Pinnacle agreed with that in Monaco within 1.5\%. The agreement in VMAT calculations between Pinnacle and Monaco using phantoms was exceptional; at the isocenter, the difference was less than $1.5 \%$ for all the patients. For independent absorbed-dose calculations, the agreement was also extremely good. For the mean dose for the PTV in particular, the agreement was within $2.0 \%$ in all the patients; specifically, no large difference was observed for high-dose regions. Conversely, a significant difference was observed in the mean dose for the OAR. For patients with prostate cancer, the mean rectal dose calculated in Monaco was significantly smaller than that calculated in Pinnacle.

Conclusions: There was no remarkable difference between the SC and XVMC calculations in the high-dose regions. The difference observed in the low-dose regions may have arisen from various causes such as the intrinsic dose deviation in the MC calculation, modeling accuracy, and CT-to-density table used in each planning system It is useful to perform independent absorbed-dose calculations with the MC algorithm in intensity-modulated radiation therapy commissioning.
\end{abstract}

\section{Introduction}

The dose delivered to a patient can be uncertain for several reasons. Dose calculations in patients using a treatment planning system (TPS) represent a main source of dose uncertainty in addition to patient setup, machine stability, and mechanical error [1,2]. The accuracy of patient

\footnotetext{
*Correspondence: haga-haga@umin.ac.jp

Department of Radiology, The University of Tokyo Hospital, Tokyo, Japan
}

dose calculations has been continuously improved by the use of the kernel-based superposition/convolution (SC) algorithm [3,4]. Recently, several commercial vendors released the Monte Carlo (MC) algorithms for photon and/or electron beam treatment planning, with the development of faster codes optimized for radiotherapy calculations and improvements in computer processing [5-8]. Consequently, the accessibility and use of MC 
treatment planning algorithms may become widespread in the radiotherapy community $[9,10]$.

By explicitly modeling the structure of the accelerator, $\mathrm{MC}$ algorithms are most credible for determining the absorbed dose, especially in the presence of heterogeneous tissue densities. This may motivate us to use an independently absorbed-dose calculation that is as accurate as the absorbed-dose calculation that was previously verified against commissioning measurements. Unlike the measurement of absorbed doses in phantoms, the independent absorbed-dose calculation using the $\mathrm{MC}$ calculation can verify the patient dose, including CT-based heterogeneity correction.

Several studies compared MC calculations with the model-based calculations [11-15]. However, less focus has been directed toward intensity-modulated radiation therapy (IMRT), in which an accurate dose calculation for irregularly shaped segments is required $[16,17]$. In principle, the added gains in accuracy for MC should be similar for volumetric modulated arc therapy (VMAT) and a multifield IMRT plan. Because of its dynamical features, it is still useful to use the MC calculation for VMAT verification to identify the cause of the beam delivery error. Almost all previous studies on VMAT verification using MC calculations have been performed for a phantom study or a specific site, and these studies have been limited to the RapidArc system using the analytical anisotropic algorithm (Varian) [18-21]. In this study, we present the result of 15 independent absorbed-dose calculations for various treatment sites in radiation therapy using Elekta VMAT created by a Pinnacle ver. 9.2 TPS (Philips). An X-ray voxel-based MC (XVMC) calculation implemented in the Monaco TPS (Elekta) was used for independent calculations. Sharing common plans, dose volume histograms (DVHs) were analyzed to quantify the dose to the targets and organ at risk (OAR). To classify the cause of the dose difference between the SC and
XVMC algorithms, the calculations were compared with the dose measurement data obtained using phantoms.

\section{Materials and methods}

Ethical consideration and consent

The use of the radiotherapy database for comprehensive and retrospective research has been approved by the committee of the Ethical Review Board in the University of Tokyo Hospital (No. 3372). This research was performed with prior written informed consent. The data was transferred into anonymous one. It makes a definite promise not to use any purpose except this research and to rigid information control.

\section{Patients and VMAT planning in Pinnacle}

Fifteen examples of the clinical treatment for patients with prostate (5 patients), lung (5 patients), head and neck (H\&N; 3 patients), rectal (1 patient), or esophageal (1 patient) cancer are presented in this study. All treatment plans were created by SmartArc in Pinnacle v9.2 (Philips, USA) with a 6-MV photon beam of a Synergy linear accelerator (Elekta) using a single arc from -179 to +179 degrees (clockwise) and $D_{95}$ prescription for the planning target volume (PTV). For the patients with prostate, $\mathrm{H} \& \mathrm{~N}$, or esophageal cancer, 2 Gy/fraction was prescribed, whereas 2.2 and $12.5 \mathrm{~Gy} /$ fraction were prescribed for the patients with rectal and lung cancers, respectively.

A control point (CP) was placed every 2 degrees, resulting in 180 CPs in a single-arc VMAT plan. The final dose calculation was performed with a grid size of $2 \mathrm{~mm}$. The collimator angle was static during rotation. The constraint on multileaf collimator (MLC) motion of $1 \mathrm{~mm} /$ degree was applied in the VMAT inverse plan for the patients with lung cancer to avoid moving the MLCs drastically during irradiation in accordance with the protocol for moving

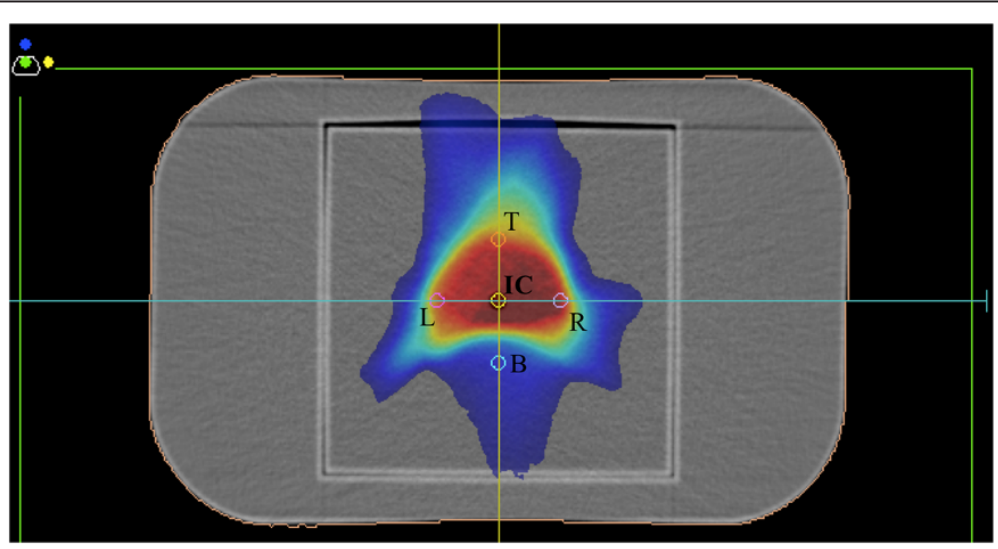

Figure 1 Computed tomographic image of the water phantom (RT-3000-New-Water, R-Tech, Japan) mapping a dose distribution. This phantom permits the insertion of a $0.6-\mathrm{mL}$ ionization chamber at the locations indicated as (isocenter), and $3 \mathrm{~cm}$ to the left and right and $3 \mathrm{~cm}$ above and below the isocenter. 
Table 1 Patient-specific doses calculated using the phantoms for the 15 patients

\begin{tabular}{|c|c|c|c|c|c|c|c|c|}
\hline & Location & Pin. (cGy) & Mon. (cGy) & Meas. (cGy) & Pin/Mon ${ }^{1}$ & Pin/Meas ${ }^{2}$ & Mon/Meas ${ }^{3}$ & SD \\
\hline \multirow[t]{5}{*}{ Prostate 1} & IC & 204.6 & 206.6 & 208.5 & 1.00 & -1.89 & -0.91 & 0.4 \\
\hline & Left & 186.3 & 185.5 & 193.1 & -0.44 & -3.55 & -3.98 & 3.9 \\
\hline & Bottom & 107.0 & 107.7 & 102.0 & 0.67 & 4.86 & 5.57 & 21.0 \\
\hline & Right & 191.1 & 189.4 & 186.4 & -0.93 & 2.56 & 1.61 & 3.8 \\
\hline & Top & 162.7 & 162.1 & 166.8 & -0.36 & -2.44 & -2.79 & 8.2 \\
\hline \multirow[t]{5}{*}{ Prostate 2} & IC & 200.5 & 201.0 & 198.9 & 0.25 & 0.83 & 1.08 & 0.4 \\
\hline & Left & 184.8 & 186.4 & 181.2 & 0.87 & 2.01 & 2.90 & 2.0 \\
\hline & Bottom & 126.5 & 118.2 & 126.1 & -6.56 & 0.30 & -6.28 & 31.6 \\
\hline & Right & 187.6 & 189.9 & 182.4 & 1.23 & 2.86 & 4.12 & 2.4 \\
\hline & Top & 159.8 & 162.9 & 150.7 & 1.94 & 6.03 & 8.09 & 9.3 \\
\hline \multirow[t]{5}{*}{ Prostate 3} & IC & 198.2 & 197.1 & 196.8 & -0.55 & 0.73 & 0.17 & 0.4 \\
\hline & Left & 175.6 & 174.7 & 177.5 & -0.51 & -1.08 & -1.59 & 4.7 \\
\hline & Bottom & 87.4 & 86.2 & 86.5 & -1.37 & 1.05 & -0.34 & 4.0 \\
\hline & Right & 189.7 & 190.7 & 185.4 & 0.53 & 2.32 & 2.86 & 3.8 \\
\hline & Top & 177.5 & 176.9 & 175.8 & -0.34 & 0.99 & 0.65 & 3.1 \\
\hline \multirow[t]{5}{*}{ Prostate 4} & IC & 207.9 & 210.6 & 208.0 & 1.30 & -0.05 & 1.25 & 0.2 \\
\hline & Left & 206.1 & 208.1 & 204.6 & 0.97 & 0.75 & 1.73 & 1.0 \\
\hline & Bottom & 71.9 & 70.0 & 70.8 & -2.64 & 1.53 & -1.15 & 19.5 \\
\hline & Right & 204.5 & 206.7 & 201.5 & 1.08 & 1.47 & 2.56 & 1.7 \\
\hline & Top & 188.1 & 190.9 & 180.5 & 1.49 & 4.18 & 5.73 & 6.5 \\
\hline \multirow[t]{5}{*}{ Prostate 5} & IC & 215.4 & 216.0 & 218.7 & 0.28 & -1.51 & -1.24 & 0.8 \\
\hline & Left & 201.1 & 201.2 & 207.5 & 0.05 & -3.10 & -3.06 & 2.3 \\
\hline & Bottom & 99.5 & 97.2 & 96.3 & -2.31 & 3.36 & 0.97 & 30.4 \\
\hline & Right & 208.4 & 208.1 & 205.1 & -0.14 & 1.62 & 1.47 & 2.4 \\
\hline & Top & 175.1 & 175.6 & 180.4 & 0.29 & -2.94 & -2.66 & 8.6 \\
\hline \multirow[t]{5}{*}{ H\&N 1} & IC & 185.3 & 186.1 & 181.4 & 0.43 & 2.17 & 2.61 & 1.5 \\
\hline & Left & 171.9 & 164.9 & 167.1 & -4.24 & 2.85 & -1.34 & 1.7 \\
\hline & Bottom & 180.5 & 178.6 & 173.6 & -1.06 & 3.99 & 2.90 & 6.2 \\
\hline & Right & 184.9 & 186.0 & 177.5 & 0.59 & 4.16 & 4.78 & 6.4 \\
\hline & Top & 171.4 & 171.9 & 166.1 & 0.29 & 3.17 & 3.47 & 3.9 \\
\hline \multirow[t]{5}{*}{ H\&N 2} & IC & 170.9 & 169.7 & 165.4 & -0.71 & 3.30 & 2.58 & 3.1 \\
\hline & Left & 175.2 & 175.7 & 168.8 & 0.28 & 3.80 & 4.10 & 3.5 \\
\hline & Bottom & 81.0 & 79.6 & 77.4 & -1.76 & 4.60 & 2.79 & 4.8 \\
\hline & Right & 171.4 & 173.3 & 171.3 & 1.10 & 0.03 & 1.14 & 0.9 \\
\hline & Top & 166.4 & 167.2 & 161.9 & 0.48 & 2.79 & 3.28 & 3.2 \\
\hline \multirow[t]{5}{*}{$H \& N 3$} & IC & 181.4 & 183.8 & 183.0 & 1.31 & -0.90 & 0.41 & 1.0 \\
\hline & Left & 124.9 & 123.4 & 133.8 & -1.22 & -6.65 & -7.77 & 11.3 \\
\hline & Bottom & 177.9 & 178.6 & 178.5 & 0.39 & -0.34 & 0.05 & 2.8 \\
\hline & Right & 142.3 & 143.5 & 127.6 & 0.84 & 11.55 & 12.49 & 6.3 \\
\hline & Top & 163.2 & 164.0 & 161.6 & 0.49 & 0.97 & 1.46 & 2.7 \\
\hline \multirow[t]{4}{*}{ Esophagus 1} & IC & 177.8 & 177.0 & 177.6 & -0.45 & 0.11 & -0.34 & 0.3 \\
\hline & Left & 183.8 & 184.2 & 183.8 & 0.22 & 0.00 & 0.22 & 0.6 \\
\hline & Bottom & 151.5 & 152.0 & 144.6 & 0.33 & 4.75 & 5.09 & 8.5 \\
\hline & Right & 161.4 & 160.0 & 156.4 & -0.88 & 3.22 & 2.32 & 3.9 \\
\hline
\end{tabular}


Table 1 Patient-specific doses calculated using the phantoms for the 15 patients (Continued)

\begin{tabular}{lcccccccc}
\hline & Top & 160.5 & 160.4 & 158.7 & $\mathbf{- 0 . 0 6}$ & $\mathbf{1 . 1 4}$ & $\mathbf{1 . 0 7}$ & 3.7 \\
Rectum 1 & IC & 211.7 & 211.1 & 211.9 & $\mathbf{- 0 . 2 8}$ & $\mathbf{- 0 . 1 0}$ & $\mathbf{- 0 . 3 8}$ & 0.9 \\
& Left & 221.2 & 221.4 & 221.8 & $\mathbf{0 . 0 9}$ & $\mathbf{- 0 . 2 7}$ & $\mathbf{- 0 . 1 8}$ & 1.4 \\
& Bottom & 197.6 & 197.4 & 197.2 & $\mathbf{- 0 . 1 0}$ & $\mathbf{0 . 2 1}$ & $\mathbf{0 . 1 1}$ & 1.2 \\
& Right & 205.9 & 207.1 & 204.6 & $\mathbf{0 . 5 8}$ & $\mathbf{0 . 6 5}$ & $\mathbf{1 . 2 3}$ & 1.2 \\
& Top & 221.2 & 215.5 & 219.3 & $\mathbf{- 2 . 6 5}$ & $\mathbf{0 . 8 7}$ & $\mathbf{- 1 . 7 3}$ & 1.4 \\
Lung 1 & IC & 1172.9 & 1179.8 & 1201.4 & $\mathbf{0 . 5 9}$ & $\mathbf{- 2 . 3 7}$ & $\mathbf{- 1 . 7 9}$ & NA \\
Lung 2 & IC & 1225.5 & 1233.6 & 1232.4 & $\mathbf{0 . 6 5}$ & $\mathbf{- 0 . 5 6}$ & $\mathbf{0 . 0 9}$ & NA \\
Lung 3 & IC & 1304.2 & 1323.5 & 1295.5 & $\mathbf{1 . 4 6}$ & $\mathbf{0 . 6 7}$ & $\mathbf{2 . 1 6}$ & NA \\
Lung 4 & IC & 1249.4 & 1265.0 & 1230.1 & $\mathbf{1 . 2 3}$ & $\mathbf{1 . 5 7}$ & $\mathbf{2 . 8 3}$ & NA \\
Lung 5 & IC & 1272.3 & 1277.1 & 1275.5 & $\mathbf{0 . 3 7}$ & $\mathbf{- 0 . 2 5}$ & $\mathbf{0 . 1 2}$ & NA \\
\hline
\end{tabular}

Five measurement volumes in the water phantom were compared for the patients with prostate, H\&N, esophageal, or rectal cancer, whereas the IC point dose in the cork phantom was compared for the patients with lung cancer. For the use of a 0.6-mL ionization chamber, the standard deviation (SD) of the dose inside the chamber evaluated by Pinnacle is indicated in the last column.

1 ([D_Pinnacle] - [D_Monaco])/[D_Pinnacle] $\times 100$.

${ }^{2}\left(\left[\mathrm{D} \_\right.\right.$Pinnacle $]-\left[\mathrm{D} \_\right.$Measurement $\left.]\right) /\left[\mathrm{D} \_\right.$Pinnacle $] \times 100$.

$3\left(\left[D \_\right.\right.$Monaco $]-\left[D \_\right.$Measurement $\left.]\right) /[$D_Monaco $] \times 100$.

targets in our hospital. On the contrary, no constraint on MLC motion was applied for the other patients.

\section{Independent absorbed-dose calculation in Monaco}

The independent absorbed-dose calculation was performed in a Monaco ver. 3.3 TPS using the XVMC code coupled with the virtual source model (VSM) ver. 1.6 based on the virtual energy fluence model of a treatment head including the primary collimator, jaws, and MLC [22,23]. The VSM was created by the vendor (Elekta) using separate internal modeling tools with numerous measurement data instead of MC transport through the detailed linac head components. After treatment planning in Pinnacle, the created plan, patient $\mathrm{CT}$, and regions of interest (ROIs) were transferred to Monaco. At the time of transfer, the VMAT plan was converted into a set of static beams in accordance with
Monaco's format of the patient QA mode by using an in-house program. In the conversion of a VMAT plan with $180 \mathrm{CPs}$, we divided the static beams into the left (from -359 to -179 degrees) and right halves (from 1 to 179 degrees) because the patient QA mode in Monaco accepts a maximum of 100 beams. In addition, the monitor unit in each beam was multiplied by 10 to avoid a rounding error in the dose calculation in Monaco. The calculated 2dose distributions were summed by the built-in function in Monaco TPS. The independent absorbed-dose calculation was performed with a grid size of $2 \mathrm{~mm}$ and a variance of $0.5 \%$ in the total beams.

\section{Evaluation}

The validation of the beam modeling for both TPSs was performed by comparing the doses for a $10-\times 10-\mathrm{cm}^{2}$

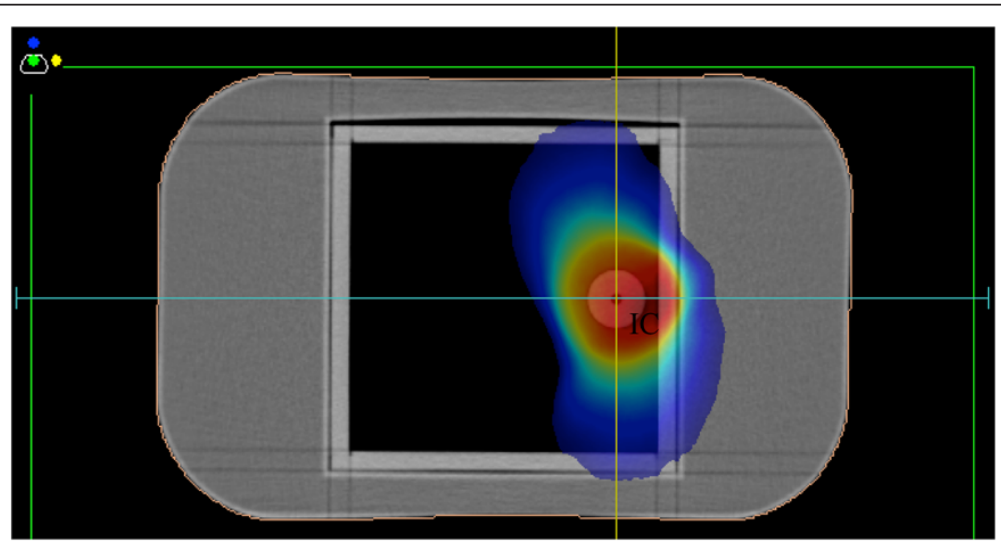

Figure 2 Computed tomographic image of a cork phantom (RT-3000-New-Water with cork, R-Tech, Japan) mapping a dose distribution. The low-density area is composed of the piled components of the cork. A spherical insert of $3 \mathrm{~cm}$ in diameter is placed anywhere inside the cork, and a $0.015-\mathrm{mL}$ pinpoint ionization chamber can be inserted. 
field irradiated from 3 different gantry angles (0, 90, and 180 degrees) using a homogeneous water phantom (RT-3000-New-Water, R-Tech, Japan) and a 0.6-mL ionization chamber (Type 30013, PTW, Germany). The locations of the measured volume were the isocenter (IC) and $3 \mathrm{~cm}$ to the left (L), $3 \mathrm{~cm}$ below (B), $3 \mathrm{~cm}$ to the right $(\mathrm{R})$, and $3 \mathrm{~cm}$ above (T) the IC (Figure 1). This 3 -cm offset provides at least 1 location at which the dose inside the ion chamber is homogeneous (meaning a relatively small standard deviation as presented in Table 1) or corresponds to that in the avoided region (e.g., the bottom in Table 1 for patients with prostate cancer is located in the rectal region).

All the treatment plans used in this study were transferred to phantoms, and the absorbed doses were calculated in both TPSs, which were compared with the measurement. The aforementioned water phantom was used for all the treatment plans, excluding those for lung cancer treatment, in which an inhomogeneous cork phantom with a spherical insert of $3 \mathrm{~cm}$ in diameter (RT-3000-New-Water with cork, R-Tech) placed on the middle of the cork and a $0.015-\mathrm{mL}$ pinpoint ionization chamber (Type 31014, PTW) were used (Figure 2). Whereas the point dose was employed for comparison with the measurement using the pinpoint chamber, the mean value of the calculated dose inside the ROI for the virtual ionization chamber was used for comparison with the measurement using the $0.6-\mathrm{mL}$ ionization chamber.

In the independent absorbed-dose calculation on the patient CT using Monaco TPS, the following set of clinically relevant dose-volume values per fraction was reported: (1) the minimal, prescription, median, mean, and maximal doses $\left(D_{\min }, D_{95}, D_{50}, D_{\text {mean }}\right.$, and $D_{\max }$, respectively) for PTV; (2) the mean and maximal doses for the rectum and bladder of the patients with prostate cancer; (3) the mean and maximal doses for the brainstem and spinal cord of the patients with $H \& N$ cancer; (4) the mean dose for both lungs and the contralateral lung of the patients with lung cancer; (5) the mean and maximal doses for the small bowel and bladder of the patients with rectal cancer; and (6) the mean and maximal doses for the spinal cord and the mean dose for the lungs of the patients with esophageal cancer.

To detect systematic differences between the dose calculation algorithms, paired $t$ tests were performed for the patients with prostate or lung cancer. A $\mathrm{P}<5 \%$ was considered statistically significant.

\section{Results}

Table 2 shows the results of the validation test using the water phantom. All the differences between the calculated value and the measurement were within $1.0 \%$. The calculated dose in Monaco TPS was in agreement with those in Pinnacle TPS. Slight differences (more than 1\%) were observed at IC and L in the irradiation with a gantry angle of 90 degrees.

The results of the patient-specific dose measurement for the 15 patients are shown in Table 1. For all of the patients, excluding those with lung cancer, 5 measurement volumes in the water phantom were compared, whereas for the patients with lung cancer, the IC point

Table 2 The results of the validation test using a water phantom

\begin{tabular}{|c|c|c|c|c|c|c|c|}
\hline & Location & Pin. (cGy) & Mon. (cGy) & Meas. (cGy) & Pin/Mon ${ }^{1}$ & Pin/Meas ${ }^{2}$ & Mon/Meas ${ }^{3}$ \\
\hline Gantry angle & IC & 153.0 & 153.6 & 153.0 & 0.39 & 0.00 & 0.39 \\
\hline 0 degrees & Left & 151.7 & 151.7 & 151.8 & 0.00 & -0.07 & -0.07 \\
\hline \multirow[t]{3}{*}{$200 \mathrm{MU}$} & Bottom & 128.9 & 130.1 & 129.0 & 0.93 & -0.08 & 0.85 \\
\hline & Right & 151.9 & 151.7 & 152.0 & -0.13 & -0.07 & -0.20 \\
\hline & Top & 180.8 & 180.5 & 180.5 & -0.17 & 0.17 & 0.00 \\
\hline Gantry angle & IC & 119.2 & 120.8 & 120.3 & 1.34 & -0.91 & 0.42 \\
\hline 90 degrees & Left & 99.7 & 101.0 & 100.0 & 1.30 & -0.30 & 1.00 \\
\hline \multirow[t]{3}{*}{$200 \mathrm{MU}$} & Bottom & 118.1 & 118.1 & 118.3 & 0.00 & -0.17 & -0.17 \\
\hline & Right & 142.5 & 142.9 & 142.4 & 0.28 & 0.07 & 0.35 \\
\hline & Top & 117.8 & 118.0 & 118.1 & 0.17 & -0.25 & -0.08 \\
\hline Gantry angle & IC & 152.0 & 152.8 & 152.5 & 0.53 & -0.33 & 0.20 \\
\hline 180 degrees & Left & 150.5 & 150.1 & 150.3 & -0.27 & 0.13 & -0.13 \\
\hline \multirow[t]{3}{*}{$200 \mathrm{MU}$} & Bottom & 179.6 & 180.3 & 180.0 & 0.39 & -0.22 & 0.17 \\
\hline & Right & 150.3 & 150.7 & 150.5 & 0.27 & -0.13 & 0.13 \\
\hline & Top & 128.1 & 129.2 & 129.0 & 0.86 & -0.70 & 0.16 \\
\hline
\end{tabular}

The comparison results are indicated as percentages in last three columns.

${ }^{1}\left(\left[D \_\right.\right.$Pinnacle] $-[$D_Monaco] $) /[$D_Pinnacle] $\times 100$.

${ }^{2}([$ D_Pinnacle $]-[$ D_Measurement $]) /[$ D_Pinnacle $] \times 100$.

$3\left(\left[D \_\right.\right.$Monaco $]-\left[D \_\right.$Measurement $\left.]\right) /\left[D \_\right.$Monaco $] \times 100$. 
Table 3 Difference between the Pinnacle SC and Monaco XVMC calculations regarding the patient CT in percentage ([D_Pinnacle] - [D_Monaco])/[D_Pinnacle] $\times$ 100)

\begin{tabular}{|c|c|c|c|c|c|c|c|c|c|}
\hline \multirow[t]{2}{*}{ Prostate } & \multicolumn{5}{|c|}{ PTV } & \multicolumn{2}{|c|}{ Rectum } & \multicolumn{2}{|c|}{ Bladder } \\
\hline & $D_{\max }$ & $D_{95}$ & $D_{50}$ & $D_{\text {mean }}$ & $D_{\min }$ & $D_{\max }$ & $\overline{D_{\text {mean }}}$ & $\underline{D}_{\max }$ & $D_{\text {mean }}$ \\
\hline 1 & 0.30 & -1.38 & -0.64 & -0.73 & -4.98 & -0.56 & -4.59 & -0.38 & 0.25 \\
\hline 2 & 3.31 & 1.72 & 0.84 & 0.94 & -2.76 & 1.37 & -4.74 & 3.67 & 5.09 \\
\hline 3 & -0.33 & -0.61 & -0.37 & -0.58 & -9.09 & -1.00 & -4.85 & 0.92 & 1.04 \\
\hline 4 & 2.79 & 1.36 & 1.41 & 1.17 & 1.07 & 1.82 & -3.18 & 1.73 & -5.73 \\
\hline 5 & 0.67 & -0.05 & 0.00 & -0.12 & -1.42 & 1.19 & -4.59 & 0.67 & 1.18 \\
\hline Mean & 1.35 & 0.21 & 0.25 & 0.14 & -3.44 & 0.56 & -4.39 & 1.32 & 0.37 \\
\hline \multirow[t]{2}{*}{$H \& N$} & \multicolumn{5}{|c|}{ PTV } & \multicolumn{2}{|c|}{ Spinal cord } & \multicolumn{2}{|c|}{ Brain stem } \\
\hline & $D_{\max }$ & $D_{95}$ & $D_{50}$ & $D_{\text {mean }}$ & $D_{\min }$ & $D_{\max }$ & $D_{\text {mean }}$ & $D_{\max }$ & $D_{\text {mean }}$ \\
\hline 1 & 1.15 & -2.99 & -0.78 & -1.28 & -6.26 & -6.31 & -11.70 & -3.56 & -21.24 \\
\hline 2 & -0.30 & -1.20 & -1.94 & -1.99 & -7.27 & 0.22 & -22.80 & 0.02 & 1.79 \\
\hline 3 & 1.60 & 1.69 & 2.03 & 1.83 & -1.94 & -3.67 & -5.77 & -1.62 & -21.19 \\
\hline Mean & 0.82 & -0.83 & -0.23 & -0.48 & -5.16 & -3.25 & -13.42 & -1.72 & -13.55 \\
\hline \multirow[t]{2}{*}{ Esophagus } & \multicolumn{5}{|c|}{ PTV } & \multicolumn{2}{|c|}{ Spinal cord } & \multicolumn{2}{|c|}{ Lung } \\
\hline & $D_{\max }$ & $D_{95}$ & $D_{50}$ & $D_{\text {mean }}$ & $D_{\min }$ & $D_{\max }$ & $D_{\text {mean }}$ & & \\
\hline 1 & 1.07 & -0.90 & -0.88 & -0.85 & -1.95 & -5.68 & -4.74 & & \\
\hline \multirow[t]{2}{*}{ Rectum } & \multicolumn{5}{|c|}{ PTV } & \multicolumn{2}{|c|}{ Small bowel } & \multicolumn{2}{|c|}{ Bladder } \\
\hline & $D_{\max }$ & $D_{95}$ & $D_{50}$ & $D_{\text {mean }}$ & $D_{\min }$ & $D_{\max }$ & $D_{\text {mean }}$ & $D_{\max }$ & $D_{\text {mean }}$ \\
\hline 1 & 1.76 & -0.55 & -0.78 & -0.78 & -0.17 & 0.62 & -2.86 & -0.17 & -2.54 \\
\hline \multirow[t]{2}{*}{ Lung } & \multicolumn{5}{|c|}{ PTV } & \multicolumn{2}{|c|}{ PTV-lung } & \multicolumn{2}{|c|}{ Contralateral lung } \\
\hline & $D_{\max }$ & $D_{95}$ & $D_{50}$ & $D_{\text {mean }}$ & $D_{\min }$ & \multicolumn{2}{|c|}{$D_{\text {mean }}$} & \multicolumn{2}{|c|}{$D_{\text {mean }}$} \\
\hline 1 & 1.30 & -0.10 & 0.57 & 0.57 & -5.50 & \multicolumn{2}{|c|}{-0.29} & \multicolumn{2}{|c|}{-4.79} \\
\hline 2 & 1.21 & -0.20 & -0.19 & -0.12 & -1.57 & \multicolumn{2}{|c|}{1.85} & \multicolumn{2}{|c|}{0.60} \\
\hline 3 & -1.34 & -0.45 & -0.24 & -0.34 & -0.67 & \multicolumn{2}{|c|}{-1.48} & \multicolumn{2}{|c|}{5.72} \\
\hline 4 & -1.79 & -0.03 & -0.45 & -0.37 & 0.19 & \multicolumn{2}{|c|}{-1.68} & \multicolumn{2}{|c|}{-2.14} \\
\hline 5 & 1.18 & -0.70 & -0.63 & -0.46 & -0.74 & & & & \\
\hline Mean & 0.11 & -0.30 & -0.19 & -0.14 & -1.66 & & & & \\
\hline
\end{tabular}

dose in the cork phantom was compared. Unlike the validation test, a relatively large difference between the calculated dose and the measurement was observed as presented in Table 1. The main cause of this difference is an inhomogeneous dose inside the $0.6-\mathrm{mL}$ virtual ionization chamber, the standard deviation of which is indicated in the last column in Table 1. We can observe that a large standard deviation, which means that the phantom location is very sensitive for the measurement, produces a relatively large difference between the calculated dose and the measurement, indicating that the measurement volume resulting in a homogeneous dose was in good agreement with the calculation. For patients with lung cancer, the patient-specific dose measurement was acceptable with the point-dose comparison. From the patient-specific QA, again it can be said that the calculated dose in Monaco TPS is in good agreement with those in Pinnacle TPS.
In Table 3, the results of the independent absorbeddose calculations for the 15 patients are summarized. There were no significant differences between the dosevolume values calculated in Monaco and Pinnacle for PTV. By contrast, a difference was observed in the mean dose for the OAR. For the patients with prostate cancer, the mean rectal dose calculated in Monaco was significantly smaller than that calculated in Pinnacle $(P=0.002)$. No significant difference was observed for the patients with lung cancer. The examples of DVH for the patients with prostate, $H \& N$, esophageal, rectal, and lung cancer are shown in Figure 3(a), (b), (c), (d), and (e), respectively.

\section{Discussion}

One of the concerns about the uncertainty in radiation therapy is how well the actual dose administrated to a patient can be reproduced in a TPS, as large deviations can be observed among the different dose calculation 

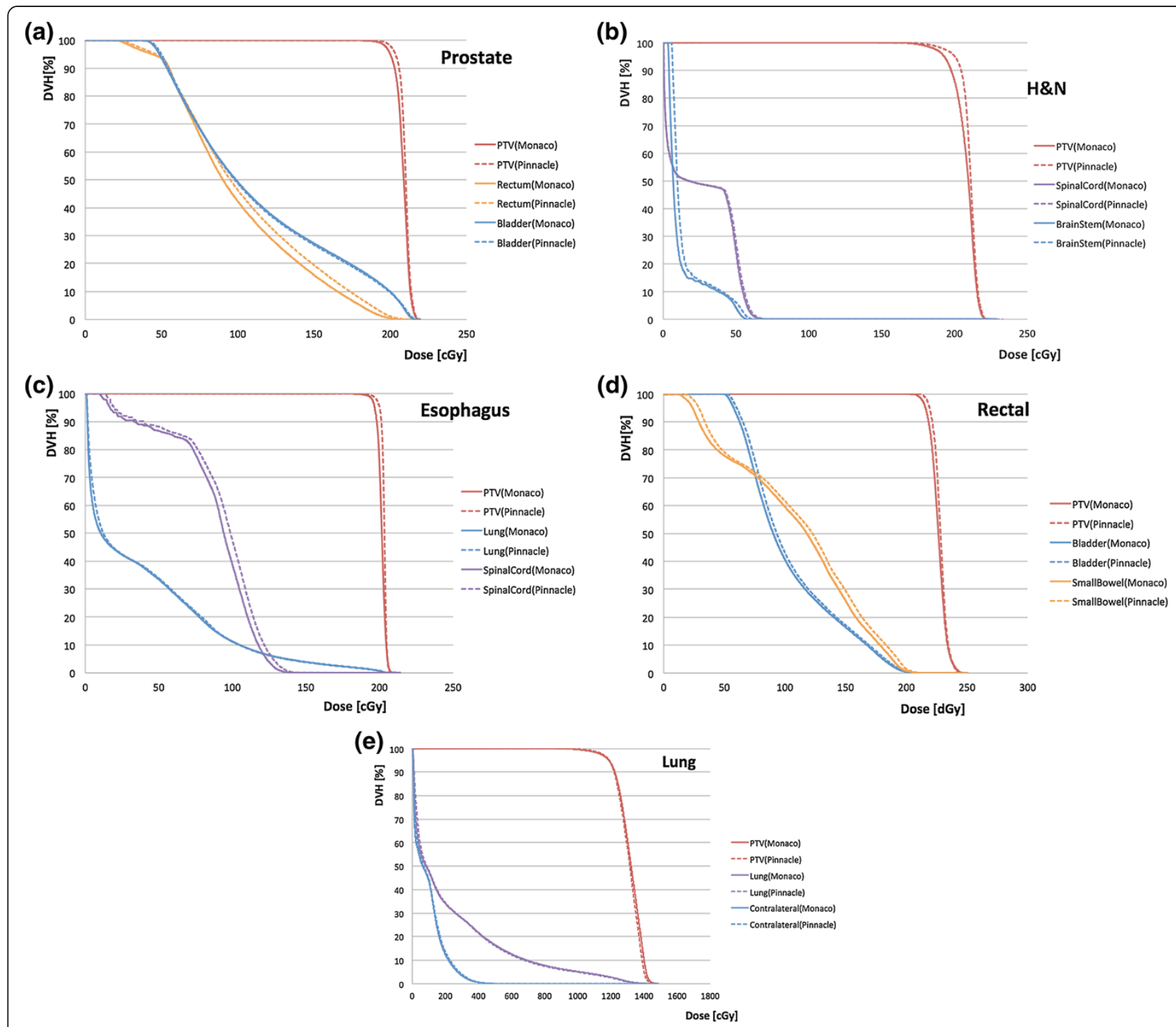

Figure 3 Dose-volume histogram (1 fraction) for the patients with (a) prostate (No. 1), (b) H\&N (No. 1), (c) esophageal, (d) rectal, or (e) lung (No. 1) cancer. The solid curves denote the Monaco DVH, whereas the dashed curves denote the Pinnacle DVH.

algorithms. In this context, the MC algorithm is expected to result in the highest dose calculation accuracy. In our independent absorbed-dose calculation using the $\mathrm{XVMC}$ algorithm, it was found that the dose difference in the PTV between the Pinnacle SC and Monaco XVMC algorithms was very small; the agreement in $D_{50}$ and $D_{\text {mean }}$ was within $2.0 \%$ in almost all the cases. The agreement for $D_{95}$ was also good, but a difference of approximately $3 \%$ difference was recorded for patient 1 with $\mathrm{H} \& \mathrm{~N}$ cancer (Figure $3[\mathrm{~b}]$ ). Some differences were observed in $D_{\min }$, for which the dose profile is likely to be steep; as a result, a slight difference in dose distribution can result in a detectable difference in $D_{\text {min }}$. In addition, the deviation in the dose calculated using the MC algorithm could affect the differences in $D_{\max }$ and $D_{\min }$. The present result confirmed a previous lung IMRT study [17], revealing that a patient dose, including lower attenuation of photon beams such as that observed in lung tissue, is adequately evaluated by the SC algorithm. In VMAT, the various beam path and build-up lengths contribute to the dose in the PTV. Consequently, the dose difference originating from various algorithms would be more moderate than the conventional IMRT when the 3-dimensional density scaling of the kernel is applied for inhomogeneous density regions, such as that used in the Pinnacle TPS. For the lung VMAT, the constraint on the MLC movement described in the Materials and Methods provided a simpler plan than that for the other sites. This finding partially explains why the $\mathrm{SC}$ calculation agreed with the $\mathrm{MC}$ calculation in the patients with lung cancer.

In contrast to the high-dose regions, a small but significant difference between the Pinnacle and Monaco 
TPSs was observed in the low-dose regions. As observed in Table 3, the doses for OARs such as the rectum in prostate cancer, the spinal cord in $\mathrm{H} \& \mathrm{~N}$ or esophageal cancer, and the small bowel in rectal cancer, as calculated by Pinnacle, tended to be overestimated compared with those calculated by the Monaco XVMC algorithm. In the water phantom study, the difference at $\mathrm{B}(3 \mathrm{~cm}$ below the IC) between Pinnacle and Monaco was similar to that in the patient study. The lower-dose regions are generally more likely to be hidden from irradiation by the MLCs. In this case, the contribution of the primary beam is relatively reduced; consequently, the accuracy of the secondary electron transport is needed in the dose calculation. For this difference, it is also possible to partially resolve this issue by adjusting the MLC transmission and leakage between leaves in either TPS. Therefore, beam remodeling is expected to improve this problem.

In general, the $\mathrm{MC}$ calculation is considered more reliable than the SC calculation. However, it should be noted that the $\mathrm{MC}$ calculation has an intrinsic deviation arising from statistical accuracy. This uncertainty is larger in low-dose regions. In fact, according to phantom studies, the result of Monaco does not always improve more than that of Pinnacle, although this finding is also influenced by measurement errors. Owing to the low-dose region, the absolute difference was small; from another point of view, it can be said that the dose in Pinnacle agreed with that in Monaco within the limit of measurement accuracy.

A discrepancy in the independent absorbed-dose calculation involves the uncertainty arising from both the beam modeling and CT-to-density curves used in TPS. Pinnacle uses the physical density as the user input, whereas Monaco uses the electron density.

The independent absorbed-dose calculation cannot replace the dosimetric verification performed routinely in patient-specific QA, which also has a role in verifying whether the created treatment plan is actually deliverable in the radiation system. As demonstrated in this study, it would be difficult to elucidate the cause of the dosimetric error from the independent dose calculation even though the MC algorithm was used. However, it does not mean that the independent calculation using the MC algorithm is illogical. The aforementioned statement is based on the assumption that the beam modeling of the TPS is perfect. It is still extremely useful to implement an independent dose calculation as a commissioning from diversified standpoints.

\section{Conclusions}

The independent absorbed-dose calculation indicated that there was no remarkable difference between the $\mathrm{SC}$ and XVMC calculations in the high-dose regions. The difference observed in the low-dose regions might have arisen from various causes, including the intrinsic dose deviation in the MC calculation, the modeling accuracy depending on the parameters such as MLC transmission and scattering, and the CT-to-density table used in each TPS. It is meaningful for IMRT commissioning to verify the modeling accuracy.

\section{Competing interests}

K.N. received the research grant from Elekta.

\section{Authors' contributions}

$\mathrm{KN}$ conceived the idea. $\mathrm{AH}, \mathrm{AS}$, and $\mathrm{AN}$ generated the treatment plan in Pinnacle. AH developed the program and drafted the manuscript. AH, ST, and $\mathrm{TI}$ calculated the dose in Monaco. AH and TM analyzed the data. ST and $\mathrm{TI}$ conducted the measurement. HI, KS, HY, KO, and KN gave the clinical advice and received the informed consents. All authors read and approved the final manuscript.

\section{Acknowledgment}

The authors would like to thank Yoshio Iwai and Kiyoshi Yoda (Elekta KK) for providing detailed information about the Monaco TPS. This work was supported by JSPS KAKENHI 22791176.

Received: 23 September 2013 Accepted: 7 March 2014

Published: 14 March 2014

\section{References}

1. Papanikolaou N, Battista J, Boyer A, Kappas C, Klein E, Mackie T, Sharpe M, Van Dyk J: Tissue Inhomogeneity Corrections for Megavoltage Photon Beams. AAPM Report No 85, Task Group No 65 of the Radiation Therapy Committee of the American Association of Physicists in Medicine. Madison, WI: Medical Physics Publishing; 2004.

2. Fraass BA, Smathers J, Deye J: Summary and recommendations of a National Cancer Institute workshop on issues limiting the clinical use of Monte Carlo dose calculation algorithms for megavoltage external beam radiation therapy. Med Phys 2003, 30:3206-3216.

3. Mackie TR, Bielajew AF, Rogers DWO, Battista JJ: Generation of photon energy deposition kernels using the EGS Monte Carlo code. Phys Med Biol 1988, 33:1-20.

4. Ahnesjö A: Collapsed cone convolution of radiant energy for photon dose calculation in heterogeneous media. Med Phys 1989, 16:577-592.

5. Chetty IJ, Curran B, Cygler JE, DeMarco JJ, Ezzell G, Faddegon BA, Kawrakow I, Keall PJ, Liu H, Ma CMC, Rogers DWO, Seuntjens J, Sheikh-Bagheri D, Siebers JV: Report of the AAPM Task Group No. 105: issues associated with clinical implementation of Monte Carlo-based photon and electron external beam treatment planning. Med Phys 2007, 34:4818-4853.

6. Kawrakow I, Fippel M, Friedrich K: 3D electron dose calculation using a voxel-based Monte Carlo algorithm (VMC). Med Phys 1996, 23:445-457.

7. Fippel M, Laub W, Huber B, Nüsslin F: Experimental investigation of a fast Monte Carlo photon beam dose calculation algorithm. Phys Med Biol 1999, 44:3039-3054.

8. Fippel M, Nüsslin F: Foundations of the Monte Carlo method for dose calculation in radiotherapy. Z Med Phys 2011, 11:73-82.

9. Fragoso M, Wen N, Kumar S, Liu D, Ryu S, Movsas B, Munther A, Chetty IJ: Dosimetric verification and clinical evaluation of a new commercially available Monte Carlo-based dose algorithm for application in stereotactic body radiation therapy (SBRT) treatment planning. Phys Med Biol 2010, 55:4445-4464.

10. Fotina I, Kragl G, Kroupa B, Trausmuth R, Georg D: Clinical comparison of dose calculation using the enhanced collapsed cone algorithm vs. a new Monte Carlo algorithm. Strahlenther Onkol 2011, 187:433-441.

11. Paelinck L, Reynaert N, Thierens H, De Neve W, De Wagter C: Experimental verification of lung dose with radiochromic film: comparison with Monte Carlo simulations and commercially available treatment planning systems. Phys Med Biol 2005, 50:2055-2069.

12. Krieger T, Sauer OA: Monte Carlo-versus pencil-beam-/collapsed cone-dose calculation in a heterogeneous multi-layer phantom. Phys Med Biol 2005, 50:859-868.

13. Fogliata A, Vanetti E, Albers D, Brink C, Clivio A, Knöös T, Nicolini G, Cozzi L: On the dosimetric behaviour of photon dose calculation algorithms in 
the presence of simple geometric heterogeneities: comparison with Monte Carlo calculations. Phys Med Biol 2007, 52:1363-1385.

14. Panettieri V, Wennberg B, Gagliardi G, Duch MA, Ginjaume M, Lax I: SBRT of lung tumours: Monte Carlo simulation with PENELOPE of dose distributions including respiratory motion and comparison with different treatment planning systems. Phys Med Biol 2007, 52:4265-4281.

15. Fotina I, Winkler P, Künzler T, Reiterer J, Simmat I, Georg D: Advanced kernel methods vs. Monte Carlo-based dose calculation for high energy photon beams. Radiother Oncol 2009, 93:645-653.

16. Crowe SB, Kairn T, Trapp JV, Feilding AL: Monte Carlo evaluation of collapsed-cone convolution calculations in head and neck radiotherapy treatment plans. World Congress on Medical Physics and Biomedical Engineering Beijing, China, IFMBE Proceedings 2013, 39:1803-1806.

17. Vanderstraeten B, Reynaert N, Paelinck L, Madani I, De Wagter C, De Gersem W, De Neve W, Thierens H: Accuracy of patient dose calculation for lung IMRT: a comparison of Monte Carlo, convolution/superposition, and pencil beam computations. Med Phys 2006, 33:3149-3158.

18. Bush K, Townson R, Zavgorodni S: Monte Carlo simulation of RapidArc radiotherapy delivery. Phys Med Biol 2008, 53:359-370.

19. Ceberg S, Gagne I, Gustafsson H, Scherman JB, Korreman SS, Kjaer-Kristoffersen F, Hilts M, Bäck SA: RapidArc treatment verification in 3D using polymer gel dosimetry and Monte Carlo simulation. Phys Med Biol 2010, 55:4885-4898.

20. Gagne Ml, Ansbacher W, Zavgorodni S, Popescu C, Beckham WA: A Monte Carlo evaluation of RapidArc dose calculations for oropharynx radiotherapy. Phys Med Biol 2008, 53:7167-7185.

21. Gete E, Duzenli C, Milette M-P, Mestrovic A, Hyde D, Bergman AM, Teke T: A Monte Carlo approach to validation of FFF VMAT treatment plans for the TrueBeam linac. Med Phys 2013, 40:021707.

22. Fippel M: Fast Monte Carlo dose calculation for photon beams based on the VMC electron algorithm. Med Phys 1999, 26:1466-1475.

23. Fippel M, Haryanto F, Dohm O, Nüsslin F, Kriesen S: A virtual photon energy fluence model for Monte Carlo dose calculation. Med Phys 2003, 30:301-311.

doi:10.1186/1748-717X-9-75

Cite this article as: Haga et al.: Independent absorbed-dose calculation using the Monte Carlo algorithm in volumetric modulated arc therapy. Radiation Oncology 2014 9:75.

\section{Submit your next manuscript to BioMed Central and take full advantage of:}

- Convenient online submission

- Thorough peer review

- No space constraints or color figure charges

- Immediate publication on acceptance

- Inclusion in PubMed, CAS, Scopus and Google Scholar

- Research which is freely available for redistribution 